Pacific

Journal of

Mathematics

\title{
A NEW UPPER BOUND \\ FOR THE DIRAC OPERATORS ON HYPERSURFACES
}

Nicolas Ginoux, Georges Habib and Simon Raulot 


\title{
A NEW UPPER BOUND \\ FOR THE DIRAC OPERATORS ON HYPERSURFACES
}

\author{
Nicolas Ginoux, Georges Habib and Simon Raulot \\ Dedicated to Oussama Hijazi for his sixtieth birthday and to Sebastián Montiel.
}

\begin{abstract}
We prove a new upper bound for the first eigenvalue of the Dirac operator of a compact hypersurface in any Riemannian spin manifold carrying a nontrivial twistor-spinor without zeros on the hypersurface. The upper bound is expressed as the first eigenvalue of a drifting Schrödinger operator on the hypersurface. Moreover, using a recent approach developed by $O$. Hijazi and S. Montiel, we completely characterize the equality case when the ambient manifold is the standard hyperbolic space.
\end{abstract}

\section{Introduction}

Let $M^{n} \stackrel{\iota}{\hookrightarrow} \widetilde{M}^{n+1}$ be an oriented, compact (without boundary), connected hypersurface of an $(n+1)$-dimensional Riemannian manifold $\left(\widetilde{M}^{n+1}, g\right)$ equipped with the induced Riemannian metric, also denoted by $g$.

It is by now a well-known approach to use the min-max characterization of eigenvalues to derive upper bounds for the spectrum of differential operators on $M$ in terms of extrinsic geometric data. For example, if we consider the first positive eigenvalue $\lambda_{1}(\Delta)$ of the Laplace operator $\Delta:=-\operatorname{tr}_{g}\left(\operatorname{Hess}_{g}\right)$, where Hess $g$ denotes the Hessian of $M$, a famous result of R.C. Reilly [1977] states that if $\widetilde{M}$ is the Euclidean space $\mathbb{R}^{n+1}$, then

$$
\lambda_{1}(\Delta) \leq \frac{n}{\operatorname{Vol}(M)} \int_{M} H^{2} d v_{g}
$$

where $H$ denotes the normalized mean curvature of $M$. The proof of this result uses, in an essential way, the Rayleigh characterization of $\lambda_{1}(\Delta)$ by choosing a modification of the coordinates functions as test functions. Moreover, it is a straightforward observation to see that equality occurs if and only if $M$ is a totally umbilical round sphere. As observed in [El Soufi and Ilias 1992], this method

MSC2010: 53C27, 53C40.

Keywords: global analysis, spectral theory, Dirac operator, geometry of submanifolds. 
directly applies for hypersurfaces in the unit sphere $\mathbb{S}^{n+1}$, leading to the counterpart of (1) in this situation:

$$
\lambda_{1}(\Delta) \leq \frac{n}{\operatorname{Vol}(M)} \int_{M}\left(H^{2}+1\right) d v_{g} .
$$

If the ambient manifold $\widetilde{M}$ is the standard hyperbolic space, there is also an optimal upper bound proved by A. El Soufi and S. Ilias [1992, Theorem 1] which improves a previous result of E. Heintze [1988] and which states that

$$
\lambda_{1}(\Delta) \leq \frac{n}{\operatorname{Vol}(M)} \int_{M}\left(H^{2}-1\right) d v_{g},
$$

with equality if and only if $M$ is a totally umbilical round sphere. All three estimates above follow actually from a much more general one, valid for submanifolds of any codimension [El Soufi and Ilias 1992], assuming solely that the ambient manifold is conformally equivalent to an open subset of the sphere of the same dimension: under that assumption,

$$
\lambda_{1}(\Delta) \leq \frac{n}{\operatorname{Vol}(M)} \int_{M}\left(H^{2}+R(\iota)\right) d v_{g},
$$

[op. cit., Theorem 2], where $R(\iota)$ is the normalized trace of the ambient sectional curvature on the tangent planes; see the precise definition after (15).

Now if we assume the existence of a spin structure on $\widetilde{M}$ (which is the case for most classical ambient spaces), it induces a spin structure on the hypersurface $M$, and so we can define the spinor bundle $\Sigma M$ over $M$ as well as the associated Dirac operator $D_{M}$ (see Section 2 and the references therein). When the ambient space $\tilde{M}$ is the space form of constant sectional curvature $\kappa \in\{0,1,-1\}$, C. Bär [1998] proved that

$$
\lambda_{1}\left(D_{M}^{2}\right) \leq \frac{n^{2}}{4 \operatorname{Vol}(M)} \int_{M}\left(H^{2}+\kappa\right) d v_{g}
$$

if $\kappa=0,1$ and

$$
\lambda_{1}\left(D_{M}^{2}\right) \leq \frac{1}{4} n^{2} \sup _{M}\left(H^{2}+1\right)
$$

for $\kappa=-1$. Here $\lambda_{1}\left(D_{M}^{2}\right)$ denotes the first nonnegative eigenvalue of the square of the Dirac operator $D_{M}$ of $(M, g)$. Those estimates are consequences of the min-max characterization of $\lambda_{1}\left(D_{M}^{2}\right)$ and the fact that the space forms $\mathbb{R}^{n+1}, \mathbb{S}^{n+1}$, and $\mathbb{R}^{n+1}$ carry, respectively, parallel, real Killing, and imaginary Killing spinors. In fact, taking the restriction of such a spinor field to the hypersurface as a test section in the Rayleigh quotient of $\lambda_{1}\left(D_{M}^{2}\right)$ gives the previous inequalities immediately. Note that these upper bounds hold for more general ambient manifolds since the proof only relies on the existence of one such particular field. For example, (5) with $\kappa=0$ 
holds for compact oriented hypersurfaces in Calabi-Yau manifolds, hyper-Kähler and some other 7- and 8-dimensional special Riemannian manifolds. It also appears that both inequalities in (5) are sharp since round geodesic spheres in the Euclidean space $\mathbb{R}^{n+1}$ and in the round sphere $\mathbb{S}^{n+1}$ satisfy the equality case. O. Hijazi and S. Montiel [2013] proved that when $\kappa=0$ those are the only hypersurfaces for which equality is achieved. The limiting case for hypersurfaces in the sphere seems to be out of reach at this time and could be considered as a spinorial analogue of the Yau conjecture about the first eigenvalue of the Laplace operator of minimal hypersurfaces in the unit sphere. However, there are nonminimal hypersurfaces in the sphere that satisfy the limiting case in (5); see, e.g., [Ginoux 2003a; Ginoux 2008].

Regarding the proof of (6), it is not difficult to observe that there are no hypersurfaces which satisfy the equality case. Modifying the computation of the Rayleigh quotient for $\lambda_{1}\left(D_{M}^{2}\right)$, this estimate can be improved [Ginoux 2003b, Theorem 1] into

$$
\lambda_{1}\left(D_{M}^{2}\right) \leq \frac{1}{4} n^{2} \sup _{M}\left(H^{2}-1\right),
$$

where equality occurs for totally umbilical round spheres in $\mathbb{H}^{n+1}$. As we will see in Corollary 4.2, those are in fact the only hypersurfaces for which (7) is an equality.

In this paper, we prove a new upper bound for the first eigenvalue of the Dirac operator of $M$ when the ambient manifold $\widetilde{M}$ carries a twistor-spinor; see Theorem 3.3. This bound coincides with the first eigenvalue of an elliptic differential operator of order two whose definition depends, among others, on the norm of the twistor-spinor along the hypersurface - see (15) - and which belongs to a particular class of operators: the drifting Schrödinger operators, that is, of the form drifting Laplacian plus potential; see Remarks 3.2. It is important to note that this estimate contains all the (up to date) known upper estimates à la Reilly; see Remarks 3.4. In a second part, we adapt the approach developed by Hijazi and Montiel [2013] to prove that, assuming the existence of imaginary Killing spinors for two opposite constants on $\tilde{M}$, the only hypersurfaces satisfying the equality case in our previous estimate are the totally umbilical ones; see Theorem 4.1. In particular, only the geodesic hyperspheres satisfy that limiting case in the hyperbolic space; see Corollary 4.2. We also examine the setting of pseudohyperbolic spaces; see Corollary 4.7.

\section{Preliminaries and notation}

In this section, we briefly introduce the geometric setting and fix the notation of this paper. For more details on those preliminaries, see examples in [Lawson and Michelsohn 1989], [Friedrich 2000], or [Ginoux 2009, Chapter 1]. 
We consider $M^{n} \stackrel{\iota}{\hookrightarrow} \widetilde{M}^{n+1}$ an oriented $n$-dimensional Riemannian hypersurface with $n \geq 2$, isometrically immersed into an $(n+1)$-dimensional Riemannian spin manifold $\left(\widetilde{M}^{n+1}, g\right)$ with a fixed spin structure. We denote by $v$ the unit inner normal vector field induced by both orientations, that is, such that $\left(E_{1}, \ldots, E_{n}, v_{x}\right)$ is an oriented basis of $\left.T_{x} \widetilde{M}\right|_{M}$ if and only if $\left(E_{1}, \ldots, E_{n}\right)$ is an oriented basis of $T_{x} M$ for $x \in M$. We endow $M$ with the spin structure induced by the one on $\widetilde{M}$ and let $\Sigma M \rightarrow M$ denotes the associated spinor bundle. Setting

$$
\Sigma:= \begin{cases}\Sigma M & \text { if } n \text { is even, } \\ \Sigma M \oplus \Sigma M & \text { if } n \text { is odd, }\end{cases}
$$

the bundles $\Sigma$ and the restriction $\left.\Sigma \tilde{M}\right|_{M}$ to $M$ of the spinor bundle of $\tilde{M}$ can be identified in such a way that:

- The natural Hermitian inner products — both of which we denote by $\langle\cdot, \cdot\rangle$ coincide.

- Clifford multiplication “." on $\tilde{M}$ and “ ${ }_{\dot{M}}$ ” on $M$ are related by

$$
X_{\dot{\Sigma}}:=X \cdot v \cdot \simeq \begin{cases}X_{\dot{M}} & \text { if } n \text { is even, } \\ X_{\dot{M}} \oplus-X_{\dot{M}} & \text { if } n \text { is odd, }\end{cases}
$$

for all $X \in T M$.

- The spin Levi-Civita connections $\widehat{\nabla}$ on $\Sigma \tilde{M}$ and $\nabla$ on $\Sigma$ are related by the spin Gauss formula

$$
\widehat{\nabla}_{X} \varphi=\nabla_{X} \varphi+\frac{1}{2} A(X) \cdot v \cdot \varphi,
$$

for all $X \in \Gamma(T M)$ and $\varphi \in \Gamma(\Sigma)$. Here $A:=-\widehat{\nabla} v$ denotes the Weingarten map of the immersion.

The extrinsic Dirac operator of $M$ is the first order elliptic differential operator of order one acting on sections of $\Sigma$ locally given by

$$
D:=\sum_{j=1}^{n} e_{j} \cdot v \cdot \nabla_{e_{j}} .
$$

It is a well-known fact that it defines an essentially self-adjoint operator with respect to the $L^{2}$-scalar product on $\Sigma$ so that if $M$ is compact, its spectrum is an unbounded sequence of real numbers. In this article, we adopt the convention that the spectrum $\operatorname{spec}(P)$ with multiplicities of a given elliptic self-adjoint operator $P$ will be denoted by a sequence $\left(\lambda_{k}(P)\right)_{k \geq 1}$, with the convention that $\lambda_{1}(P)$ is the smallest eigenvalue if $\operatorname{spec}(P)$ is bounded below and is the smallest nonnegative eigenvalue otherwise.

With respect to the previous identifications, the Dirac operator $D$ is nothing but the Dirac operator $D_{M}$ of $(M, g)$ if $n$ is even and $D_{M} \oplus-D_{M}$ if $n$ is odd, so that 
studying the spectrum of the intrinsic Dirac operator $D_{M}$ for the spin Riemannian structure induced on the hypersurface $M$ is equivalent to study the spectrum of the extrinsic Dirac operator $D$ on the hypersurface $M$. It is also relevant here to recall that the commutator of $D$ and $D^{2}$ with functions are given by

$$
D(f \varphi)=f D \varphi+\nabla f \cdot v \cdot \varphi
$$

and

$$
D^{2}(f \varphi)=f D^{2} \varphi-2 \nabla_{\nabla f} \varphi+(\Delta f) \varphi,
$$

for all $f \in C^{\infty}(M)$ and $\varphi \in \Gamma(\Sigma)$. Here $H:=\frac{1}{n} \operatorname{tr}(A)$ denotes the mean curvature function of $M$ in $\widetilde{M}$.

Another operator of particular interest in this work is the Dirac-Witten operator $\hat{D}$ on $M$. It is also a first order elliptic operator acting on the restricted spinor bundle $\Sigma$ and locally defined by $\hat{D}:=\sum_{j=1}^{n} e_{j} \cdot \widehat{\nabla}_{e_{j}}$. It is related to the extrinsic Dirac operator by the following formula

$$
D \varphi=-v \cdot \hat{D} \varphi+\frac{1}{2} n H \varphi
$$

and to its square by

$$
D^{2} \varphi=\hat{D}^{2} \varphi+\frac{1}{4} n^{2} H^{2} \varphi+\frac{1}{2} n \nabla H \cdot v \cdot \varphi,
$$

for every $\varphi \in \Gamma(\Sigma)$.

\section{Upper bounds in terms of a Laplace-type operator}

In this section, we prove a new upper bound for the smallest eigenvalue of the squared Dirac operator $D^{2}$ when the ambient manifold $\tilde{M}$ is endowed with a twistorspinor. Recall that a twistor-spinor on a Riemannian spin manifold $\left(\widetilde{M}^{n+1}, g\right)$ is a section $\psi \in \Gamma(\Sigma \tilde{M})$ satisfying

$$
\widehat{\nabla}_{X} \psi=-\frac{1}{n+1} X_{\dot{\tilde{M}}} D_{\tilde{M}} \psi
$$

for all $X \in \Gamma(T \tilde{M})$. Here $D_{\widetilde{M}}$ represents the Dirac operator of $\tilde{M}$. Nonzero twistorspinors have a discrete vanishing set and only exist for particular conformal classes; see, for example, the standard reference [Baum et al. 1991] or, for a short account, [Ginoux 2009, Appendix A]. It should also be pointed out that parallel spinors, and real and imaginary Killing spinors are twistor-spinors which are, in addition, eigensections for the Dirac operator $D_{\widetilde{M}}$ associated to the eigenvalue zero, or to real or purely imaginary eigenvalues, respectively. They exist on each simply connected complete space form of constant curvature. Assume now that such a spinor field $\psi$ is given on $\widetilde{M}$ and also assume that it has no zero on the hypersurface $M$. We 
define the differential operator $L_{\psi}$ acting on smooth functions on $M$ by

$$
L_{\psi} f:=\Delta f-2 g(\nabla \ln |\psi|, \nabla f)+\frac{1}{4} n^{2}\left(H^{2}+R(\iota)\right) f .
$$

for $f \in C^{\infty}(M)$. Here

$$
R(\iota):=\frac{1}{n(n-1)}(\tilde{S}-2 \widetilde{\operatorname{ric}}(v, v)),
$$

$\tilde{S}$ and $\tilde{\text { ric }}$ are respectively the scalar curvature and the Ricci tensor (seen as a symmetric 2-tensor) of the manifold $\tilde{M}$. Although this operator is not symmetric with respect to the $L^{2}$-scalar product on $\left(M^{n}, g\right)$, we observe that it has the following interesting analytic properties:

Proposition 3.1. The operator $L_{\psi}$ is elliptic, and if $M$ is closed, it is self-adjoint with respect to the $L^{2}$-scalar product on $\left(M^{n}, \bar{g}\right)$, where $\bar{g}:=|\psi|^{4 / n} g$.

Proof. Since $L_{\psi}$ is of second order and its leading part is the scalar Laplacian, it is clearly elliptic. Because $\bar{g}=|\psi|^{4 / n} g$, we have $d v_{\bar{g}}=|\psi|^{2} d v_{g}$ and for any $f, h \in C^{\infty}(M)$,

$$
\int_{M}\left(L_{\psi} f\right) h d v_{\bar{g}}=\int_{M}\left(\Delta f-2 g(\nabla \ln |\psi|, \nabla f)+\frac{1}{4} n^{2}\left(H^{2}+R(\iota)\right) f\right) h|\psi|^{2} d v_{g} .
$$

Performing a partial integration, we have for the first term

$$
\begin{aligned}
\int_{M}(\Delta f) h|\psi|^{2} d v_{g} & =\int_{M} g(\nabla f, \nabla h)|\psi|^{2}+g\left(\nabla f, \nabla\left(|\psi|^{2}\right)\right) h d v_{g} \\
& =\int_{M} g(\nabla f, \nabla h)|\psi|^{2}+2 g(\nabla f, \nabla \ln |\psi|) h|\psi|^{2} d v_{g} .
\end{aligned}
$$

Therefore, the first-order term in $\nabla \ln |\psi|$ simplifies and we obtain

$$
\int_{M}\left(L_{\psi} f\right) h d v_{\bar{g}}=\int_{M}\left(g(\nabla f, \nabla h)+\frac{1}{4} n^{2}\left(H^{2}+R(\iota)\right) f h\right)|\psi|^{2} d v_{g},
$$

which is clearly symmetric in $(f, h)$. This implies that $L_{\psi}$ is formally self-adjoint with respect to the metric $\bar{g}$. Since $M$ is closed, we conclude that $L_{\psi}$ is essentially self-adjoint in $L^{2}(M)$.

Remarks 3.2. (1) The operator $L_{\psi}$ defined in (15) is of the form drifting Laplacian plus potential (the drifting Laplacian is also called Laplacian with drift, BakryÉmery Laplacian, weighted Laplacian, or Witten Laplacian in the literature); this is the reason we refer to these operators as drifting Schrödinger operators. Indeed, a drifting Laplacian is an operator of the form

$$
C^{\infty}(M) \stackrel{L_{h}}{\longrightarrow} C^{\infty}(M), \quad f \mapsto \Delta f-g(\nabla h, \nabla f)
$$

for some function $h \in C^{\infty}(M)$. It is elliptic and self-adjoint with respect to the measure $e^{h} d \mu_{g}$. Actually, a drifting Laplacian is always unitarily equivalent to a 
Schrödinger operator: in the notation above, the operator $L_{h}$ is unitarily equivalent to $\Delta-\frac{1}{2} \Delta h+\frac{1}{4}|\nabla h|_{g}^{2}$; see, for example, [Setti 1998, p. 28].

(2) Note that if $|\psi|$ is constant on $M$ (which is the case if $\psi$ is either a parallel or a real Killing spinor on $\widetilde{M}$ ), then the operator

$$
L_{\psi}=\Delta+\frac{1}{4} n^{2}\left(H^{2}+R(\iota)\right)
$$

does not depend on $\psi$.

Proposition 3.1 implies that the spectrum of $L_{\psi}$ is purely discrete. We will denote by $\lambda_{1}\left(L_{\psi}\right)$ its first eigenvalue, which satisfies the min-max characterization

$$
\lambda_{1}\left(L_{\psi}\right)=\inf _{f \in C^{\infty}(M) \backslash\{0\}}\left(\frac{\int_{M} f\left(L_{\psi} f\right) d v_{\bar{g}}}{\int_{M} f^{2} d v_{\bar{g}}}\right) .
$$

We are now ready to give the precise statement of the first main result of this paper, namely:

Theorem 3.3. Assume $M$ is a closed oriented hypersurface isometrically immersed in a Riemannian spin manifold $\left(\widetilde{M}^{n+1}, g\right)$. If there exists a nontrivial twistor-spinor $\psi$ on $\tilde{M}$ with $\psi_{x} \neq 0$ for all $x \in M$, then

$$
\lambda_{1}\left(D_{M}^{2}\right) \leq \lambda_{1}\left(L_{\psi}\right) .
$$

Proof. We apply the min-max characterization of $\lambda_{1}\left(D_{M}^{2}\right)=\lambda_{1}\left(D^{2}\right)$ using $f \psi$ as a test section, where $L_{\psi} f=\lambda_{1}\left(L_{\psi}\right) f$. The following computations rely on a large extent on those in the proof of [Ginoux 2009, Theorem 5.2.3].

First, if $f \in C^{\infty}(M)$ is an arbitrary smooth function on $M$, then using (11), (13), and (9) and the fact that $\psi$ is a twistor-spinor on $\tilde{M}$,

$$
\begin{aligned}
D^{2}(f \psi) \stackrel{(11)}{=} & f D^{2} \psi-2 \nabla_{\nabla f} \psi+(\Delta f) \psi \\
\stackrel{(13)}{=} f( & \left.\hat{D}^{2} \psi+\frac{1}{4} n^{2} H^{2} \psi+\frac{1}{2} n \nabla H \cdot v \cdot \psi\right) \\
& \quad-2 \nabla_{\nabla f} \psi+(\Delta f) \psi \\
\stackrel{(9)}{=} & f\left(\hat{D}^{2} \psi+\frac{1}{4} n^{2} H^{2} \psi+\frac{1}{2} n \nabla H \cdot v \cdot \psi\right) \\
& \quad-2\left(\widehat{\nabla}_{\nabla f} \psi-\frac{1}{2} A(\nabla f) \cdot v \cdot \psi\right)+(\Delta f) \psi \\
= & f\left(\hat{D}^{2} \psi+\frac{1}{4} n^{2} H^{2} \psi+\frac{1}{2} n \nabla H \cdot v \cdot \psi\right)+\frac{2}{n+1} \nabla f \cdot D_{\widetilde{M}} \psi \\
& +A(\nabla f) \cdot v \cdot \psi+(\Delta f) \psi .
\end{aligned}
$$

Next we compute $\hat{D}^{2} \psi$, again using the fact that $\psi$ is a twistor-spinor, which implies, in particular, the following identity; see, e.g., [Ginoux 2009, Proposition A.2.1]:

$$
\widehat{\nabla}_{X}\left(D_{\widetilde{M}} \psi\right)=\frac{n+1}{n-1}\left(-\frac{1}{2} \widetilde{\operatorname{Ric}}(X) \cdot \psi+\frac{1}{4 n} \tilde{S} X \cdot \psi\right),
$$


for every $X \in \Gamma(T \widetilde{M})$ and where Ric denotes the Ricci tensor of $\left(\widetilde{M}^{n+1}, g\right)$ (seen as an endomorphism of the tangent bundle of $\tilde{M})$. Thus we have

$$
\begin{aligned}
\hat{D}^{2} \psi & =\hat{D}\left(\sum_{j=1}^{n} e_{j} \cdot \widehat{\nabla}_{e_{j}} \psi\right) \\
& \stackrel{(14)}{=} \frac{n}{n+1} \hat{D}\left(D_{\tilde{M}} \psi\right) \\
& \stackrel{(19)}{=} \frac{n}{n-1} \sum_{j=1}^{n}\left(-\frac{1}{2} e_{j} \cdot \widetilde{\operatorname{Ric}}\left(e_{j}\right) \cdot \psi+\frac{1}{4 n} \tilde{S} e_{j} \cdot e_{j} \cdot \psi\right) \\
& =\frac{n}{n-1}\left(\frac{1}{2} \tilde{S} \psi+\frac{1}{2} v \cdot \widetilde{\operatorname{Ric}}(v) \cdot \psi-\frac{1}{4} \tilde{S} \psi\right) \\
& =\frac{n}{n-1}\left(\frac{n(n-1)}{4} R(\iota) \psi+\frac{1}{2} v \cdot \widetilde{\operatorname{Ric}}(v)^{\top} \cdot \psi\right) \\
& =\frac{1}{4} n^{2} R(\iota) \psi+\frac{n}{2(n-1)} v \cdot \widetilde{\operatorname{Ric}}(v)^{\top} \cdot \psi
\end{aligned}
$$

where $\widetilde{\operatorname{Ric}}(v)^{\top}:=\sum_{j=1}^{n} \widetilde{\operatorname{ric}}\left(v, e_{j}\right) e_{j}$ denotes the tangential projection of $\widetilde{\operatorname{Ric}}(v)$ on TM. Combining (18) with (20), we deduce that

$$
\begin{aligned}
D^{2}(f \psi)=\frac{1}{4} n^{2}\left(H^{2}+R(\iota)\right) f \psi & +\frac{1}{2} n f \nabla H \cdot v \cdot \psi+\frac{n}{2(n-1)} f v \cdot \widetilde{\operatorname{Ric}}(v)^{\top} \cdot \psi \\
& +\frac{2}{n+1} \nabla f \cdot D \widetilde{M} \psi+A(\nabla f) \cdot v \cdot \psi+(\Delta f) \psi
\end{aligned}
$$

Again, using the fact that $\psi$ is a twistor-spinor on $\left(\tilde{M}^{n+1}, g\right)$, for every $f \in C^{\infty}(M)$,

$$
\begin{aligned}
\operatorname{Re}\left\langle D^{2}(f \psi), f \psi\right\rangle \stackrel{(21)}{=} \frac{1}{4} n^{2}\left(H^{2}+R(\iota)\right) f^{2}|\psi|^{2}+\frac{2}{n+1} f \operatorname{Re}\langle\nabla f \cdot D \widetilde{M} \psi, \psi\rangle & \\
& \quad+f(\Delta f)|\psi|^{2} \\
= & \frac{1}{4} n^{2}\left(H^{2}+R(\iota)\right) f^{2}|\psi|^{2}-g\left(f \nabla f, \nabla\left(|\psi|^{2}\right)\right) \\
& \quad+f(\Delta f)|\psi|^{2} \\
= & f\left(\Delta f-2 g(\nabla f, \nabla \ln |\psi|)+\frac{1}{4} n^{2}\left(H^{2}+R(\iota)\right) f\right)|\psi|^{2} \\
= & f\left(L_{\psi} f\right)|\psi|^{2} .
\end{aligned}
$$

The min-max principle for $\lambda_{1}\left(D^{2}\right)$ implies that, for any $f \in C^{\infty}(M) \backslash\{0\}$,

$$
\lambda_{1}\left(D^{2}\right) \leq \frac{\int_{M} \operatorname{Re}\left\langle D^{2}(f \psi), f \psi\right\rangle d v_{g}}{\int_{M}|f \psi|^{2} d v_{g}}=\frac{\int_{M} f\left(L_{\psi} f\right) d v_{\bar{g}}}{\int_{M} f^{2} d v_{\bar{g}}} ;
$$

therefore,

$$
\lambda_{1}\left(D^{2}\right) \leq \inf _{f \in C^{\infty}(M, \mathbb{R}) \backslash\{0\}}\left(\frac{\int_{M} f\left(L_{\psi} f\right) d v_{\bar{g}}}{\int_{M} f^{2} d v_{\bar{g}}}\right),
$$

which from (16) gives the inequality (17). 
Remarks 3.4. (1) The estimate (17) contains all known upper estimates à la Reilly for $\lambda_{1}\left(D_{M}^{2}\right)$. Indeed, we observe that by taking $f=1$ in the Rayleigh quotient of $L_{\psi}$,

$$
\lambda_{1}\left(L_{\psi}\right) \leq \frac{n^{2}}{4 \operatorname{Vol}(M)} \int_{M}\left(H^{2}+R(\iota)\right) d v_{g}
$$

if $|\psi|$ is constant, and

$$
\lambda_{1}\left(L_{\psi}\right) \leq \frac{1}{4} n^{2} \sup _{M}\left(H^{2}+R(\iota)\right)
$$

otherwise. Those give exactly the inequalities (5) in [Bär 1998] and (7) in [Ginoux 2003b]. On the other hand, for $f=|\psi|^{-1}$ (with respect to the metric $\bar{g}$ defined above), we deduce that

$$
\lambda_{1}\left(L_{\psi}\right) \leq \frac{n^{2}}{4 \operatorname{Vol}(M)} \int_{M}\left(H^{2}+R(\iota)\right) d v_{g}+\frac{1}{\operatorname{Vol}(M)} \int_{M}|d \ln | \psi||^{2} d v_{g},
$$

which was proved in [Ginoux 2002, Theorem 1].

(2) It is interesting to compare (17) with (4). On the one hand, we do not obtain in the spinorial setting the exact analogue of (4) for $\widetilde{M}$ conformally equivalent to an open subset of the sphere $\mathbb{S}^{n+1}$. Of course, this must be expected since otherwise in dimension 2 this would mean that the Willmore functional bounds $\lambda_{1}\left(D_{M}^{2}\right) \cdot \operatorname{Area}\left(M^{2}, g\right)$ from above; but there is no conformal upper bound for the smallest positive Dirac eigenvalue on unit-area-metrics, as shown in [Ammann and Jammes 2012, Theorem 1.1]. Note that this does not prevent the analogue of (3) to possibly hold true for the Dirac operator, which is still an open question. On the other hand, our assumption on $\widetilde{M}$ in Theorem 3.3 is much more general since not only open subsets of spheres with conformal metrics allow twistor-spinors. We refer the reader to [Kühnel and Rademacher 1998] for the classification of Riemannian spin manifolds with twistor-spinors.

We now look at the equality case of the previous estimate in the situation where the twistor-spinor is also an eigenspinor for the Dirac operator of $\widetilde{M}$. More precisely, we prove:

Proposition 3.5. Under the same assumptions as in Theorem 3.3, assume moreover that equality is achieved in (17).

(1) If $\psi$ is a parallel spinor on $\widetilde{M}^{n+1}$, then

$$
A(\nabla \ln |f|)=-\frac{1}{2} n \nabla H
$$

for any eigenfunction $f$ of $L_{\psi}$ associated with $\lambda_{1}\left(L_{\psi}\right)$. 
(2) If $\psi$ is a real Killing spinor on $\tilde{M}=\mathbb{S}^{n+1}$ or an imaginary Killing spinor on $\widetilde{M}=\llbracket^{n+1}$, then the mean curvature $H$ is constant and, in particular,

$$
\lambda_{1}\left(D_{M}^{2}\right)=\frac{1}{4} n^{2}\left(H^{2}+\kappa\right) .
$$

Proof. (1) If (17) is an equality and $\psi$ is a parallel spinor, then the min-max principle yields $D^{2}(f \psi)=\lambda_{1}\left(D^{2}\right) f \psi$ for any eigenfunction $f$ of $L_{\psi}$ associated with $\lambda_{1}\left(L_{\psi}\right)=\lambda_{1}\left(D^{2}\right)$. But (21) together with $\widetilde{\text { Ric }}=0$ and $D_{\widetilde{M}} \psi=0$ (both provided by $\widehat{\nabla} \psi=0$ ) implies

$$
\begin{aligned}
\lambda_{1}\left(D^{2}\right) f \psi & =\frac{1}{4} n^{2} H^{2} f \psi+\frac{1}{2} n f \nabla H \cdot v \cdot \psi+A(\nabla f) \cdot v \cdot \psi+(\Delta f) \psi \\
& =\left(L_{\psi} f\right) \psi+\left(A(\nabla f)+\frac{1}{2} n f \nabla H\right) \cdot v \cdot \psi .
\end{aligned}
$$

With $\lambda_{1}\left(D^{2}\right)=\lambda_{1}\left(L_{\psi}\right)$, we deduce that

$$
\left(A(\nabla f)+\frac{1}{2} n f \nabla H\right) \cdot v \cdot \psi=0
$$

which, since $\psi \neq 0$, gives $A(\nabla f)+\frac{1}{2} n f \nabla H=0$. Since any eigenfunction for $L_{\psi}$ associated with the eigenvalue $\lambda_{1}\left(L_{\psi}\right)$ is either positive or negative, we easily reach the conclusion.

(2) Assume first that $\tilde{M}^{n+1}$ carries real Killing spinors and let $\psi$ be a nonzero $(\varepsilon / 2)$-Killing spinor for some $\varepsilon \in\{ \pm 1\}$; that is, $\widehat{\nabla}_{X} \psi=\frac{\varepsilon}{2} X \cdot \psi$ for all $X \in \Gamma(T \tilde{M})$. Again, one obtains $D^{2}(f \psi)=\lambda_{1}\left(D^{2}\right) f \psi$ for any eigenfunctions $f \in C^{\infty}(M)$ associated to $\lambda_{1}\left(L_{\psi}\right)$. Fixing such an $f$, the identity (21) yields

$$
\lambda_{1}\left(D^{2}\right) f \psi=\left(L_{\psi} f\right) \psi+\left(A(\nabla f)+\frac{1}{2} n f \nabla H\right) \cdot v \cdot \psi-\varepsilon \nabla f \cdot \psi .
$$

With $\lambda_{1}\left(D^{2}\right)=\lambda_{1}\left(L_{\psi}\right)$, we deduce that

$$
\left(A(\nabla f)+\frac{1}{2} n f \nabla H\right) \cdot v \cdot \psi-\varepsilon \nabla f \cdot \psi=0 .
$$

In particular, with the notation $Y_{\varepsilon}:=-\varepsilon \nabla f$ and $X:=A(\nabla f)+\frac{1}{2} n f \nabla H$, we have $\left(Y_{\varepsilon}+X \wedge v\right) \cdot \psi=0$. At this point, we need the following claim:

Claim: Let $\alpha \in \Lambda^{*} \mathbb{R}^{n+1} \otimes \mathbb{C}$. If $n$ is odd, then $\delta_{n+1}(\alpha)=0$ if and only if $\alpha=0$. If $n$ is even, then the same equivalence holds for $\alpha \in \wedge^{*} \mathbb{R}^{n} \otimes \mathbb{C}$.

Proof of Claim. Recall that the spinor representation $\delta_{k}: \mathbb{C l}_{k} \longrightarrow \operatorname{End}_{\mathbb{C}}\left(\Sigma_{k}\right)$ of the complex Clifford algebra in dimension $k$ is a complex-linear isomorphism for $k$ even (but obviously not for $k$ odd). So if $n$ is odd, the claim follows directly from this fact. If $n$ is even and $\alpha \in \wedge^{*} \mathbb{R}^{n} \otimes \mathbb{C}$, then $\Sigma_{n} \cong \Sigma_{n+1}$ and it is a simple trick to rewrite $\delta_{n+1}(\alpha)$ in the form $\delta_{n}(\check{\alpha})$ for a form $\check{\alpha} \in \Lambda^{*} \mathbb{R}^{n} \otimes \mathbb{C}$ having the same coefficients as $\alpha$ in the canonical basis of $\Lambda^{*} \mathbb{R}^{n} \otimes \mathbb{C}$, up to sign and some power of $i$. Namely, write

$$
\alpha=\sum_{1 \leq j_{1}<\cdots<j_{k} \leq n} \alpha_{j_{1}, \ldots, j_{k}} e_{j_{1}}^{*} \wedge \cdots \wedge e_{j_{k}}^{*},
$$


where $\left(e_{1}, \ldots, e_{n}, e_{n+1}\right)$ is the canonical basis of $\mathbb{R}^{n+1}$. Let $\omega_{n}^{\mathbb{C}}$ denotes the complex volume form on $\mathbb{R}^{n}$ as defined in the proof of Proposition 3.5, which acts on $\Sigma_{n}$ via $\delta_{n}\left(\omega_{n}^{\mathbb{C}}\right)=\operatorname{Id}_{\Sigma_{n}^{+}} \oplus-\operatorname{Id}_{\Sigma_{n}^{-}}$. Since, for all $v \in \mathbb{R}^{n}, \delta_{n+1}\left(i e_{n+1}\right)=\delta_{n}\left(\omega_{n}^{\mathbb{C}}\right)$ and $\delta_{n}(v)=\delta_{n+1}(v) \circ \delta_{n+1}\left(e_{n+1}\right)$, after some calculation,

$$
\begin{aligned}
& \delta_{n+1}(\alpha)=\sum_{\substack{k \text { even } \\
1 \leq j_{1}<\cdots<j_{k} \leq n}} \alpha_{j_{1}, \ldots, j_{k}} \delta_{n}\left(e_{j_{1}}\right) \circ \cdots \circ \delta_{n}\left(e_{j_{k}}\right) \\
& +i \sum_{\substack{k \text { odd } \\
1 \leq j_{1}<\cdots<j_{k} \leq n}} \alpha_{j_{1}, \ldots, j_{k}} \delta_{n}\left(e_{j_{1}}\right) \circ \cdots \circ \delta_{n}\left(e_{j_{k}}\right) \circ \delta_{n}\left(\omega_{n}^{\mathbb{C}}\right) .
\end{aligned}
$$

Now it is an elementary computation to show that, for any $\beta \in \bigwedge^{k} \mathbb{R}^{n}$,

$$
\delta_{n}(\beta) \circ \delta_{n}\left(e_{1}^{*} \wedge \cdots \wedge e_{n}^{*}\right)=(-1)^{k(k+1) / 2} \delta_{n}(* \beta),
$$

where $*: \wedge^{*} \mathbb{R}^{n} \rightarrow \wedge^{*} \mathbb{R}^{n}$ is the Hodge-star operator. Therefore, we obtain

$$
\begin{aligned}
& \delta_{n+1}(\alpha)=\sum_{\substack{k \text { even } \\
1 \leq j_{1}<\cdots<j_{k} \leq n}} \alpha_{j_{1}, \ldots, j_{k}} \delta_{n}\left(e_{j_{1}}\right) \circ \cdots \circ \delta_{n}\left(e_{j_{k}}\right) \\
& +c_{n, k} \sum_{\substack{k \text { odd } \\
1 \leq j_{1}<\cdots<j_{k} \leq n}} \alpha_{j_{1}, \ldots, j_{k}} \delta_{n}\left(*\left(e_{j_{1}}^{*} \wedge \cdots \wedge e_{j_{k}}^{*}\right)\right)=\delta_{n}(\check{\alpha}),
\end{aligned}
$$

where we let $c_{n, k}:=i^{n / 2+1}(-1)^{k(k+1) / 2}$ and

$$
\check{\alpha}:=\sum_{\substack{k \text { even } \\ 1 \leq j_{1}<\cdots<j_{k} \leq n}} \alpha_{j_{1}, \ldots, j_{k}} e_{j_{1}}^{*} \wedge \cdots \wedge e_{j_{k}}^{*}+c_{n, k} \sum_{\substack{k \text { odd } \\ 1 \leq j_{1}<\cdots<j_{k} \leq n}} \alpha_{j_{1}, \ldots, j_{k}} *\left(e_{j_{1}}^{*} \wedge \cdots \wedge e_{j_{k}}^{*}\right) \text {. }
$$

As a consequence, if $\delta_{n+1}(\alpha) \sigma=0$ for all $\sigma \in \Sigma_{n+1} \cong \Sigma_{n}$, then $\delta_{n}(\check{\alpha})=0$, and the fact mentioned above implies $\check{\alpha}=0$; since $n$ is even, each form $*\left(e_{j_{1}}^{*} \wedge \cdots \wedge e_{j_{k}}^{*}\right)$ is of odd degree when $k$ is odd and therefore $\alpha_{j_{1}, \ldots, j_{k}}=0$ for all $1 \leq j_{1}<\cdots<j_{k} \leq n$; that is, $\alpha=0$. This concludes the proof of the claim.

If $\widetilde{M}^{n+1}$ is isometric to the standard round sphere $\mathbb{S}^{n+1}$, then it carries a maximal number (that is $2^{\left\lfloor\frac{n+1}{2}\right\rfloor}$ ) of linearly independent $(\varepsilon / 2)$-Killing spinors. In that case, $\left(Y_{\varepsilon}+X \wedge \nu\right) \cdot \psi=0$ holds pointwise for every $\psi \in \Sigma_{x} \tilde{M}$. If $n$ is odd, then the claim yields $Y_{\varepsilon}+X \wedge v=0$, which implies $X=Y_{\varepsilon}=0$; that is, $f$ and $H$ are constant. If $n$ is even, then one may rewrite

$$
\left.Y_{\varepsilon} \cdot \psi+X \cdot v \cdot \psi=i Y_{\varepsilon} \cdot i v \cdot v \cdot \psi+X \cdot v \cdot \psi=\left(X-i Y_{\varepsilon}\right\lrcorner \omega_{M}^{\mathbb{C}}\right) \cdot v \cdot \psi,
$$

where $\omega_{M}^{\mathbb{C}}:=i^{\left.i \frac{n+1}{2}\right\rfloor} e_{1}^{*} \wedge \cdots \wedge e_{n}^{*} \in \Gamma\left(\wedge^{n} T^{*} M \otimes \mathbb{C}\right)$ is the complex volume form on $M$. Again, the claim yields $\left.X-i Y_{\varepsilon}\right\lrcorner \omega_{M}^{\mathbb{C}}=0$. If $n>2$, then comparing the degrees yields $X=Y_{\varepsilon}=0$; that is, $f$ and $H$ are constant. If $n=2$, then an elementary computation gives $Z\lrcorner \omega_{M}^{\mathbb{C}}=i J(Z)$ for every $Z \in \Gamma(T M)$, where $J$ is the Kähler structure associated to the metric and the orientation on $\left(M^{2}, g\right)$. In 
that case, one obtains $X+J\left(Y_{\varepsilon}\right)=0$. However on the standard sphere $\mathbb{S}^{3}$, both spaces of $\pm \frac{1}{2}$-Killing spinors have maximal dimension 2, therefore $X+J\left(Y_{\varepsilon}\right)=0$ for both $\varepsilon \in\{ \pm 1\}$, which implies $X=Y_{\varepsilon}=0$ and hence that $f$ and $H$ are constant.

The case of imaginary Killing spinors is much the same up to replacing $\varepsilon$ by $i \varepsilon$. One obtains at the end $\left(i Y_{\varepsilon}+X \wedge v\right) \cdot \psi=0$ for all $(i \varepsilon / 2)$-Killing spinors $\psi$ on $\widetilde{M}^{n+1}$. The same arguments as above lead to $X=Y_{\varepsilon}=0$. Notice that in the case $n=2$, one does not need the existence of maximal spaces of $(i \varepsilon / 2)$-Killing spinors for both $\varepsilon \in\{ \pm 1\}$ since $X$ and $Y_{\varepsilon}$ are real vector fields on $M$.

Remark 3.6. It is quite surprising that in the case where $\psi$ is a parallel spinor we cannot conclude that the mean curvature of $M$ must be constant. In fact, we are left to prove that if there exists a smooth positive function $f \in C^{\infty}(M)$ such that

$$
\Delta f+\frac{1}{4} n^{2} H^{2} f=\lambda_{1}(D)^{2} f \quad \text { and } \quad A(\nabla \ln f)=-\frac{1}{2} n \nabla H,
$$

then $f$ (or, equivalently, $H$ ) is constant on $M$.

\section{The equality case in the presence of imaginary Killing spinors}

In this section, we focus on the equality case of our estimate (17) when the ambient manifold $\widetilde{M}$ carries an imaginary Killing spinor. According to Proposition 3.5, it also corresponds to the equality case of the inequality (7). It is obvious to check that totally umbilical round spheres in the hyperbolic space $\mathbb{H}^{n+1}$ satisfy the equality in this estimate; however, it is still unknown if they are the only ones. In fact, if the hypersurface is embedded, then this result easily follows from the Alexandrov theorem in the hyperbolic space; see [Montiel 1999]. However, if the hypersurface is only assumed to be immersed, then the question is still open. In order to settle this problem, we adopt a method introduced in [Hijazi and Montiel 2013] which relies on the fact that such hypersurfaces are critical points for some eigenvalue functional associated to some Dirac-type operator on $M$. The main result of this section concerns the case when $\widetilde{M}=\mathbb{U}^{n+1}$ but actually we will prove the following more general statement:

Theorem 4.1. Let $M^{n}$ be an oriented, compact, connected hypersurface immersed in a Riemannian spin manifold $\left(\widetilde{M}^{n+1}, g\right)$. If $\widetilde{M}$ carries an $(i \varepsilon / 2)$-Killing spinor for some $\varepsilon \in\{ \pm 1\}$, then (7) (as well as (17)) holds and if equality holds then the

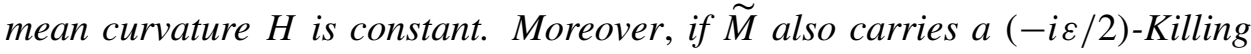
spinor, then equality holds if and only if $M$ is totally umbilical with constant mean curvature.

Since the standard hyperbolic space $\mathbb{\boxplus}^{n+1}$ has both $(i / 2)$ - and $(-i / 2)$-Killing spinors (see, e.g., [Baum 1989a]), the previous result immediately implies the next: 
Corollary 4.2. The only oriented, compact, connected hypersurfaces immersed into the hyperbolic space $\mathbb{H}^{n+1}$ satisfying $\lambda_{1}\left(D_{M}^{2}\right)=\frac{1}{4} n^{2}\left(H^{2}-1\right)$ are the totally umbilical round spheres.

In Section 4D, we will discuss the case of pseudohyperbolic spaces.

4A. The Hijazi-Montiel approach in the presence of imaginary Killing spinors. Assume that the ambient manifold $\tilde{M}$ carries an (i/2)-Killing spinor $\Psi \in \Gamma(\Sigma \tilde{M})$. After restriction to $M$, it is a straightforward computation to show that $\Psi$ satisfies the modified Dirac equation

$$
D_{+} \Psi=\frac{n}{2} H \Psi
$$

where $D_{+}$is a zero order modification of the extrinsic Dirac operator defined by

$$
D_{+} \varphi:=D \varphi-\frac{n}{2} i \nu \cdot \varphi
$$

for $\varphi \in \Gamma(\Sigma)$. Note that we do not assume that the mean curvature $H$ is constant for the moment. Suppose however that $H$ is positive everywhere on $M$, and consider the metric conformally related to $g$ on $M$, defined by $\bar{g}:=H^{2} g$. It is a well-known fact [Hitchin 1974; Hijazi 1986] that under a conformal change of the metric, there exists a bundle isometry $\varphi \mapsto \bar{\varphi}, \Sigma \rightarrow \bar{\Sigma}$, between the two extrinsic spinor bundles $\Sigma$ and $\bar{\Sigma}$ over $\left(M^{n}, g\right)$ and $\left(M^{n}, \bar{g}\right)$. Under this identification, the extrinsic Dirac operators $D$ and $D^{H}$ associated to $g$ and $\bar{g}$ and acting respectively on $\Sigma$ and $\bar{\Sigma}$ are related by

$$
D^{H} \bar{\varphi}=H^{-(n+1) / 2} \overline{D\left(H^{(n-1) / 2} \varphi\right)}
$$

for all $\varphi \in \Gamma(\Sigma)$. Now consider on $\bar{\Sigma}$ the zero order modification of the extrinsic Dirac operator $D^{H}$ given by

$$
D_{+}^{H} \bar{\varphi}:=D^{H} \bar{\varphi}-\frac{n}{2} H^{-1} \mathcal{I}_{\nu} \bar{\varphi}
$$

where $\mathcal{I}_{\nu}$ is the Hermitian endomorphism of $\bar{\Sigma}$ defined by $\mathcal{I}_{\nu} \bar{\varphi}:=\overline{i \nu \cdot \varphi}$ for all $\varphi \in \Gamma(\Sigma)$. Notice that $D_{+}^{H}$ is an elliptic and self-adjoint differential operator of order one which, since $M$ is assumed to be compact, has a discrete spectrum. In the following, we will denote by $\lambda_{1}\left(D_{+}^{H}\right)$ the first nonnegative eigenvalue of $D_{+}^{H}$. Now for every $\varphi \in \Gamma(\Sigma)$, consider the spinor field $\varphi_{H}:=H^{-(n-1) / 2} \varphi \in \Gamma(\Sigma)$ which is easily seen to satisfy

$$
D_{+}^{H} \bar{\varphi}_{H}=H^{-(n+1) / 2} \overline{D_{+} \varphi},
$$

using the conformal covariance (24) of $D$. Using (22) on the (i/2)-Killing spinor $\Psi \in \Gamma(\Sigma \tilde{M})$ in the previous identity gives $D_{+}^{H} \bar{\Psi}_{H}=\frac{n}{2} \bar{\Psi}_{H}$. This immediately implies that $\lambda_{1}\left(D_{+}^{H}\right) \leq \frac{n}{2}$. Furthermore, if the mean curvature $H$ is constant, it is 
an easy computation using $\{D, i v \cdot\}=0$ to show that

$$
\operatorname{Spec}\left(\left(D_{+}^{H}\right)^{2}\right)=\left\{\lambda_{k}\left(\left(D_{+}^{H}\right)^{2}\right)=\frac{H^{-2}\left(\lambda_{k}(D)^{2}+\frac{1}{4} n^{2}\right)}{\lambda_{k}(D)} \in \operatorname{Spec}(D)\right\},
$$

so that $\lambda_{1}\left(D_{+}^{H}\right)=\frac{n}{2}$ if and only if $\lambda_{1}\left(D^{2}\right)=\frac{n^{2}}{4}\left(H^{2}-1\right)$. Thus, we have proved this:

Proposition 4.3. Let $M$ be an orientable, compact, connected hypersurface immersed in a Riemannian spin manifold $\left(\tilde{M}^{n+1}, g\right)$ admitting a $(i / 2)$-Killing spinor, and suppose that the mean curvature of $M$, after a suitable choice of the unit normal, satisfies $H>0$. Then the first nonnegative eigenvalue of $D_{+}^{H}$ satisfies $\lambda_{1}\left(D_{+}^{H}\right) \leq \frac{n}{2}$. Moreover, if $H$ is constant, equality occurs if and only if equality occurs in (7).

From this proposition, we deduce that any immersion for which (7) - or equivalently (17) - is an equality realizes a maximum for the map

$$
\mathcal{F}_{1}^{+}: \operatorname{Imm}^{+}(M, \tilde{M}) \rightarrow \mathbb{R}, \quad \iota \mapsto \lambda_{1}\left(D_{+}^{H_{\iota}}\right),
$$

where $\operatorname{Imm}^{+}(M, \tilde{M})$ denotes the space of isometric immersions of $M$ in $\tilde{M}$ with nonvanishing mean curvature $H_{l}$. This characterization of hypersurfaces satisfying the equality case in (7) leads to the study of the critical points of the functional $\mathcal{F}_{1}^{+}$. Remark 4.4. If the manifold $\tilde{M}$ carries a ( $-i / 2)$-Killing spinor, then Proposition 4.3 is true with the operators $D_{+}$and $D_{+}^{H}$ replaced respectively by

$$
D_{-}:=D+\frac{n}{2} i v \cdot: \Gamma(\Sigma) \rightarrow \Gamma(\Sigma)
$$

and

$$
D_{-}^{H}:=D^{H}+\frac{n}{2} H^{-1} \mathcal{I}_{v}: \Gamma(\bar{\Sigma}) \rightarrow \Gamma(\bar{\Sigma}) .
$$

In this situation, the corresponding functional is defined by

$$
i \mathcal{F}_{1}^{-}: \iota \mapsto \lambda_{1}^{-}\left(D_{-}^{H_{\iota}}\right)
$$

where $\lambda_{1}^{-}\left(D_{-}^{H_{\iota}}\right)$ is the first nonnegative eigenvalue of $D_{-}^{H_{\iota}}$.

4B. Derivatives of the functional $\mathcal{F}_{1}^{ \pm}$. As explained in the previous section we are led to study the first derivatives of the functional $\mathcal{F}_{1}^{ \pm}$at least in a particular situation. As above, we start with an immersion $\iota=\iota_{0}: M \rightarrow \widetilde{M}$ with positive mean curvature (not necessarily constant) and such that $\lambda_{1}\left(D_{+}^{H}\right)=\frac{n}{2}$. Note that here we do not assume the existence of imaginary Killing spinor fields on $\widetilde{M}$.

Now we deform the immersion $\iota$ along normal geodesics; that is, we consider, for $\varepsilon>0$ sufficiently small, the map $F:]-\varepsilon, \varepsilon\left[\times M \rightarrow \tilde{M},(t, x) \mapsto \exp _{\iota(x)}\left(t v_{x}\right)\right.$. Note that, choosing $\varepsilon>0$ sufficiently small, the map $F$ is smooth and $F(t, \cdot): M \rightarrow \widetilde{M}$ is an immersion such that $F(0, \cdot)=\iota$. In fact, the map $t \mapsto F(t, x)$ is the geodesic 
starting from $\iota(x)$ with speed vector $v_{x}$, and so it is analytic. For each $\left.t \in\right]-\varepsilon, \varepsilon[$, we denote by $g_{t}:=F(t, \cdot)^{*} g$ the induced metric on $M$, by $v_{t}$ the unit normal field inducing the orientation of $M$, by $H_{t}:=-\frac{1}{n} \operatorname{tr}\left(\widehat{\nabla} v_{t}\right)$ the mean curvature of $F(t, \cdot)-$ which, up to making $\varepsilon>0$ smaller, may be assumed to be positive on $M$ for all $t \in]-\varepsilon, \varepsilon\left[-\right.$ and we set $\bar{g}_{t}:=H_{t}^{2} g_{t}$.

We also denote by $D^{H_{t}}$ the Dirac operator associated to the metric $\bar{g}_{t}$ and let $D_{+}^{H_{t}}:=D^{H_{t}}-\frac{n}{2} H_{t}^{-1} \mathcal{I}_{v_{t}}: \Gamma\left(\bar{\Sigma}_{t}\right) \rightarrow \Gamma\left(\bar{\Sigma}_{t}\right)$, where $\mathcal{I}_{v_{t}}$ is the Hermitian endomorphism of $\bar{\Sigma}_{t}$ defined by $\mathcal{I}_{v_{t}} \bar{\varphi}:=\overline{i v_{t} \cdot \varphi}$. Here $\bar{\Sigma}_{t}$ denotes the extrinsic spinor bundle over $M$ endowed with the spin structure induced by $\widetilde{M}$ and the Riemannian metric $\bar{g}_{t}$. Since we perturb the immersion analytically, the family $\left(D_{+}^{H_{t}}\right)$ with $\left.t \in\right]-\varepsilon, \varepsilon[$ is an analytic family of unbounded closed self-adjoint operators with compact resolvent, therefore the spectrum of $D_{+}^{H_{t}}$ can be written as a sequence $\left(\mu_{k}^{+}(t)\right)_{k \in \mathbb{N}}$, where each eigenvalue $\mu_{k}^{+}(t)$ depends analytically on $t$ and where corresponding eigenvectors can be found to also depend analytically on $t$; see [Kato 1995]. We denote by $\lambda_{1}^{+}(t)$ any branch of that spectrum with $\lambda_{1}^{+}(0)=\lambda_{1}\left(D_{+}^{H}\right)$, the smallest nonnegative eigenvalue of $D_{+}^{H}=D_{+}^{H_{0}}$. Following [Bär et al. 2005], we denote by $\tau_{0}^{t}: \bar{\Sigma}_{0}=\bar{\Sigma} \rightarrow \bar{\Sigma}_{t}$ the parallel transport along the curves $s \mapsto(s, x)$ in the so-called generalized cylinder (]$-\varepsilon, \varepsilon\left[\times M, d t^{2} \oplus \bar{g}_{t}\right)$, for all $\left.t \in\right]-\varepsilon, \varepsilon[$. Then for any analytic family $\left(\bar{\Phi}_{t}\right)_{t}$ of eigenvectors associated to $\lambda_{1}^{+}(t)$, differentiating the identity

$$
\lambda_{1}^{+}(t) \int_{M}\left|\bar{\Phi}_{t}\right|^{2} d v_{\bar{g}_{t}}=\int_{M} \operatorname{Re}\left\langle D_{+}^{H_{t}} \bar{\Phi}_{t}, \bar{\Phi}_{t}\right\rangle d v_{\bar{g}_{t}}
$$

at $t=0$ yields

$$
\frac{d \lambda_{1}^{+}}{d t}(0) \int_{M}\left|\bar{\Phi}_{0}\right|^{2} d v_{\bar{g}_{0}}=\int_{M} \operatorname{Re}\left\langle\left.\frac{d}{d t}\right|_{t=0}\left(\tau_{t}^{0} D_{+}^{H_{t}} \tau_{0}^{t} \bar{\Phi}_{0}\right), \bar{\Phi}_{0}\right\rangle d v_{\bar{g}_{0}} .
$$

Now we have $\tau_{t}^{0} D_{+}^{H_{t}} \tau_{0}^{t}=\tau_{t}^{0} D^{H_{t}} \tau_{0}^{t}-\frac{n}{2} H_{t}^{-1} \tau_{t}^{0} \mathcal{I}_{v_{t}} \tau_{0}^{t}$ and, since the variation of $\iota$ is a geodesic normal one, the vector field $v_{t}=\frac{\partial}{\partial t}$ is parallel along the curves $s \mapsto(s, x)$, so that $\tau_{t}^{0} \mathcal{I}_{v_{t}} \tau_{0}^{t}=\mathcal{I}_{v_{0}}=\mathcal{I}_{\nu}$ for all $\left.t \in\right]-\varepsilon, \varepsilon$ [. With the formula for the first variation of the Dirac operator by J.-P. Bourguignon and P. Gauduchon [1992] (see also [Bär et al. 2005]), we deduce that

$$
\begin{aligned}
\frac{d \lambda_{1}^{+}}{d t}(0) & \int_{M}\left|\bar{\Phi}_{0}\right|^{2} d v_{\bar{g}_{0}} \\
= & -\frac{1}{2} \int_{M} \bar{g}_{0}\left(T_{\bar{\Phi}_{0}}, \frac{\partial \bar{g}_{t}}{\partial t}(0)\right) d v_{\bar{g}_{0}}+\left.\frac{n}{2} \int_{M} H^{-2} \frac{\partial H_{t}}{\partial t}\right|_{t=0} \operatorname{Re}\left\langle\mathcal{I}_{\nu} \bar{\Phi}_{0}, \bar{\Phi}_{0}\right\rangle d v_{\bar{g}_{0}},
\end{aligned}
$$

where

$$
T_{\bar{\Phi}_{0}}(X, Y):=\frac{1}{2} \operatorname{Re}\left\langle X_{\dot{\Sigma}}^{-} \bar{\nabla}_{Y} \bar{\Phi}_{0}+Y_{\dot{\Sigma}} \bar{\nabla}_{X} \bar{\Phi}_{0}, \bar{\Phi}_{0}\right\rangle
$$

is the so-called energy-momentum tensor associated to $\bar{\Phi}_{0}$. Here $\bar{\Sigma}_{\bar{\Sigma}}$ is the Clifford multiplication on $\bar{\Sigma}$ defined by (8) and $\bar{\nabla}$ is the spin Levi-Civita connection with 
respect to the metric $\bar{g}_{0}$. Note that we kept the same notation for the Hermitian scalar products on $\bar{\Sigma}$ and $\Sigma$. Now fix an eigenvector $\bar{\Phi}_{0} \in \Gamma(\bar{\Sigma})$ for the Dirac-type operator $D_{+}^{H}$ associated with $\lambda_{1}\left(D_{+}^{H}\right)$ and let $\bar{\Psi}_{0}:=H^{(n-1) / 2} \bar{\Phi}_{0}$. We compute $d \lambda_{1}^{+} / d t(0)$ in terms of $\Psi_{0} \in \Gamma(\Sigma)$ and of geometric quantities attached to $\iota$. First, since $\partial F / \partial t(0, \cdot)=v$, we have on the one hand (see [Montiel 1999])

$$
\frac{\partial \bar{g}_{t}}{\partial t}(0)=\left.\frac{\partial}{\partial t}\right|_{t=0}\left(H_{t}^{2} g_{t}\right)=\frac{1}{n} 2 H\left(|A|^{2}+\widetilde{\operatorname{ric}}(v, v)\right) g-2 H^{2} g(A \cdot, \cdot) .
$$

On the other hand, using the isomorphism $\Sigma \rightarrow \bar{\Sigma}$, we may write (see, e.g., [Ginoux 2009, Section 1.3])

$$
T_{\bar{\Phi}_{0}}(X, Y)=H^{-n+2} T_{\Psi_{0}}(X, Y)
$$

for all $X, Y \in \Gamma(T M)$, where $T_{\Psi_{0}}$ is the energy-momentum tensor associated to $\Psi_{0}$ defined by

$$
T_{\Psi_{0}}(X, Y):=\frac{1}{2} \operatorname{Re}\left\langle X_{\dot{\Sigma}} \nabla_{Y} \Psi_{0}+Y_{\dot{\Sigma}} \nabla_{X} \Psi_{0}, \Psi_{0}\right\rangle
$$

Therefore, assuming without loss of generality that $\int_{M}\left|\bar{\Phi}_{0}\right|^{2} d v_{\bar{g}_{0}}=1$, we compute

$$
\begin{aligned}
\frac{d \lambda_{1}^{+}}{d t}(0)=\frac{1}{n} \int_{M} H^{-1}\left(|A|^{2}+\widetilde{\operatorname{ric}}(\nu, v)\right)\left(\frac{n}{2} \operatorname{Re}\left\langle i v \cdot \Psi_{0}, \Psi_{0}\right\rangle-\right. & \left.g\left(T_{\Psi_{0}}, g\right)\right) d v_{g} \\
& +\int_{M} g\left(T_{\Psi_{0}}, A\right) d v_{g} .
\end{aligned}
$$

But since $g\left(T_{\Psi_{0}}, g\right)=\operatorname{tr}_{g}\left(T_{\Psi_{0}}\right)=\operatorname{Re}\left\langle D \Psi_{0}, \Psi_{0}\right\rangle$, we obtain

$$
\frac{d \lambda_{1}^{+}}{d t}(0)=-\frac{1}{n} \int_{M} H^{-1}\left(|A|^{2}+\widetilde{\operatorname{ric}}(v, v)\right) \operatorname{Re}\left\langle D_{+} \Psi_{0}, \Psi_{0}\right\rangle d v_{g}+\int_{M} g\left(T_{\Psi_{0}}, A\right) d v_{g} .
$$

However, since $\bar{\Phi}_{0} \in \Gamma(\bar{\Sigma})$ is an eigenspinor for $D_{+}^{H}$ associated with the eigenvalue $\lambda_{1}^{+}(0)=\frac{n}{2}$ and from the equivalence

$$
D_{+}^{H} \bar{\Phi}_{0}=\frac{n}{2} \bar{\Phi}_{0} \quad \Longleftrightarrow \quad D_{+} \Psi_{0}=\frac{n}{2} H \Psi_{0}
$$

one concludes that

$$
\frac{d \lambda_{1}^{+}}{d t}(0)=-\frac{1}{2} \int_{M}\left(|A|^{2}+\widetilde{\operatorname{ric}}(v, v)\right)\left|\Psi_{0}\right|^{2} d v_{g}+\int_{M} g\left(T_{\Psi_{0}}, A\right) d v_{g} .
$$

To compute the remaining term $g\left(T_{\Psi_{0}}, A\right)$, we define a new covariant derivative by $\widehat{\nabla}_{X}^{+}:=\widehat{\nabla}_{X}-(i / 2) X \cdot$ on $\Sigma$. Then a lengthy but direct calculation using the spin 
Gauss formula (9) yields that for any $\varphi \in \Gamma(\Sigma)$,

$$
\begin{aligned}
\left|\widehat{\nabla}^{+} \varphi\right|^{2} & :=\sum_{j=1}^{n}\left|\widehat{\nabla}_{e_{j}}^{+} \varphi\right|^{2} \\
& =\sum_{j=1}^{n}\left|\nabla_{e_{j}} \varphi+\frac{1}{2} A\left(e_{j}\right) \cdot v \cdot \varphi-\frac{i}{2} e_{j} \cdot \varphi\right|^{2} \\
& =|\nabla \varphi|^{2}+\frac{1}{4}\left(|A|^{2}+n\right)|\varphi|^{2}-g\left(T_{\varphi}, A\right)-\operatorname{Re}\left\langle i v \cdot\left(D \varphi-\frac{1}{2} n H \varphi\right), \varphi\right\rangle .
\end{aligned}
$$

For $\varphi=\Psi_{0}$, we deduce, using the right-hand side of (26), that

$$
g\left(T_{\Psi_{0}}, A\right)=\left|\nabla \Psi_{0}\right|^{2}-\left|\widehat{\nabla}^{+} \Psi_{0}\right|^{2}+\frac{1}{4}\left(|A|^{2}-n\right)\left|\Psi_{0}\right|^{2} .
$$

Now integrating this identity over $M$ with the help of the famous SchrödingerLichnerowicz formula

$$
D^{2}=\nabla^{*} \nabla+\frac{1}{4} S
$$

gives

$$
\begin{aligned}
\int_{M} g\left(T_{\Psi_{0}}, A\right) & d v_{g} \\
= & \int_{M}\left(\operatorname{Re}\left\langle D^{2} \Psi_{0}, \Psi_{0}\right\rangle-\frac{1}{4} S\left|\Psi_{0}\right|^{2}-\left|\widehat{\nabla}^{+} \Psi_{0}\right|^{2}+\frac{1}{4}\left(|A|^{2}-n\right)\left|\Psi_{0}\right|^{2}\right) d v_{g} .
\end{aligned}
$$

Here $S$ stands for the scalar curvature of $\left(M^{n}, g\right)$. On the other hand, from (10), (26), and the anticommutativity rule $\{D, i v \cdot\}=0^{+}$, we check that

$$
D^{2} \Psi_{0}=\frac{1}{4} n^{2}\left(H^{2}-1\right) \Psi_{0}+\frac{1}{2} n \nabla H \cdot v \cdot \Psi_{0},
$$

so that $\operatorname{Re}\left\langle D^{2} \Psi_{0}, \Psi_{0}\right\rangle=\frac{1}{4} n^{2}\left(H^{2}-1\right)\left|\Psi_{0}\right|^{2}$, and hence

$$
\int_{M} g\left(T_{\Psi_{0}}, A\right) d v_{g}=\int_{M}\left(\frac{1}{4}\left(n^{2}\left(H^{2}-1\right)-S+|A|^{2}-n\right)\left|\Psi_{0}\right|^{2}-\left|\widehat{\nabla}^{+} \Psi_{0}\right|^{2}\right) d v_{g} .
$$

The Gauss formula for the scalar curvature provides

$$
S=\tilde{S}-2 \tilde{\operatorname{ric}}(v, v)+n^{2} H^{2}-|A|^{2},
$$

from which

$$
\begin{aligned}
& \int_{M} g\left(T_{\Psi_{0}}, A\right) d v_{g} \\
& \quad=-\int_{M}\left(\frac{1}{4}(\tilde{S}+n(n+1))-\frac{1}{2}\left(|A|^{2}+\tilde{\operatorname{ric}}(\nu, v)\right)\right)\left|\Psi_{0}\right|^{2} d v_{g}-\int_{M}\left|\widehat{\nabla}^{+} \Psi_{0}\right|^{2} d v_{g}
\end{aligned}
$$

follows. Inserting this identity in (27), we finally deduce that

$$
\frac{d \lambda_{1}^{+}}{d t}(0)=-\int_{M}\left(\left|\widehat{\nabla}^{+} \Psi_{0}\right|^{2}+\frac{1}{4}(\tilde{S}+n(n+1))\left|\Psi_{0}\right|^{2}\right) d v_{g} .
$$


It is worth noticing that this formula holds if we assume that it is the first nonnegative eigenvalue $\lambda_{1}\left(D_{-}^{H}\right)$ of $D_{-}^{H}$ which satisfies $\lambda_{1}\left(D_{-}^{H}\right)=\frac{n}{2}$ instead of $\lambda_{1}\left(D_{+}^{H}\right)$; in this situation, $\widehat{\nabla}^{+}$has to be replaced with the covariant derivative defined by $\widehat{\nabla}_{X}^{-}:=\widetilde{\nabla}_{X}+\frac{i}{2} X$

From this computation, it is now straightforward to give a necessary condition for an immersion $\iota$ to be a critical point of $\mathcal{F}_{1}^{ \pm}$:

Theorem 4.5. Let $M$ be an oriented, compact, connected hypersurface isometrically immersed in a Riemannian spin manifold $\left(\widetilde{M}^{n+1}, g\right)$. Assume that the scalar curvature $\tilde{S}$ of $\tilde{M}$ is greater or equal to $-n(n+1)$ and that the mean curvature $H$ of $M$ with respect to a suitable choice of the normal is positive. If $\lambda_{1}\left(D_{\varepsilon}^{H}\right)=\frac{n}{2}$ for some $\varepsilon \in\{ \pm 1\}$ and it is critical for all the variations of the hypersurface $M$ in $\tilde{M}$, then $\tilde{S}=-n(n+1)$ and $\widetilde{\nabla}_{X} \Psi=\frac{i \varepsilon}{2} X \cdot \Psi$ for all $X \in \Gamma(T M)$ for all $\Psi \in \Gamma(\Sigma)$ satisfying

$$
D_{\varepsilon} \Psi=\frac{n}{2} H \Psi .
$$

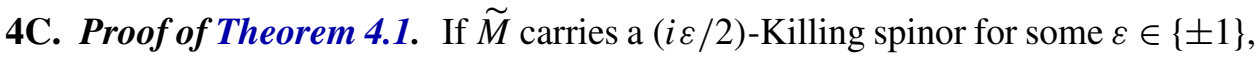
then from Theorem 3.3 and Remarks 3.4, the inequalities (17) and (7) hold. Moreover, if equality holds in (17), then Proposition 3.5 implies that the mean curvature is constant and then $\lambda_{1}(D)^{2}=\frac{1}{4} n^{2}\left(H^{2}-1\right)$.

Assume now that $\tilde{M}$ carries an $(i / 2)$ - as well as a $(-i / 2)$-Killing spinor. From Proposition 4.3, we deduce that such an immersion is a maximum for the functional $\mathcal{F}_{1}^{+}$and thus $d \lambda_{1}^{+} / d t(0)=0$. Let $\Phi$ be a nonzero $(-i / 2)$-Killing spinor on $\tilde{M}$ so that $D_{-} \Phi=\frac{n}{2} H \Phi$. From this equation and since $H$ is constant, a direct computation shows that the spinor $\tilde{\Phi}:=H \Phi-i \nu \cdot \Phi$ satisfies $D_{+} \tilde{\Phi}=\frac{n}{2} H \tilde{\Phi}$. On the other hand, since the existence of a $( \pm i / 2)$-Killing spinor on $\widetilde{M}$ implies that $\widetilde{M}$ is an Einstein manifold with scalar curvature $\tilde{S}=-n(n+1)$ (see [Baum et al. 1991], for example), Theorem 4.5 applies and we get that $\widehat{\nabla}_{X} \tilde{\Phi}=\frac{i}{2} X \cdot \tilde{\Phi}$ for all $X \in \Gamma(T M)$; that is,

$$
\begin{aligned}
\frac{i}{2} X \cdot(H \Phi-i v \cdot \Phi) & =\widehat{\nabla}_{X}(H \Phi-i v \cdot \Phi) \\
& =H\left(-\frac{i}{2} X \cdot \Phi\right)+i A(X) \cdot \Phi-i v \cdot\left(-\frac{i}{2} X \cdot \Phi\right) \\
& =i A(X) \cdot \Phi-\frac{i}{2} H X \cdot \Phi-\frac{i}{2} X \cdot i \nu \cdot \Phi .
\end{aligned}
$$

This implies that $(A(X)-H X) \cdot \Phi=0$ for all $X \in \Gamma(T M)$, and since $\Phi$ has no zero, $M$ is totally umbilical. This concludes the proof of Theorem 4.1.

4D. The case of pseudohyperbolic spaces. In this section, we examine the case of other complete ambient manifolds $\widetilde{M}$ carrying imaginary Killing spinors. These manifolds have been classified by H. Baum [1989b; 1989a] and are known as pseudohyperbolic spaces. For the sake of completeness and since we need an additional argument for our purpose, we recall the result of those references and give a sketch of the proof: 
Proposition 4.6. Let $\left(\widetilde{M}^{n+1}, g\right)$ be a complete Riemannian spin manifold admitting a nonzero (i $\varepsilon / 2)$-Killing spinor for some $\varepsilon \in\{ \pm 1\}$. Then $\left(\widetilde{M}^{n+1}, g\right)$ is isometric to either the real hyperbolic space of constant sectional curvature -1 or to the warped product $\left(\mathbb{R} \times N, d t^{2} \oplus e^{2 t} g_{N}\right)$, where $\left(N^{n}, g_{N}\right)$ is a complete nonflat Riemannian spin manifold carrying at least one nonzero parallel spinor. In the latter case, if $n$ is odd, denote by $\mathcal{K}_{0}\left(N, g_{N}\right)$ the space of parallel spinors on $\left(N^{n}, g_{N}\right)$ for the induced metric and spin structure, and if $n$ is even, denote by $\mathcal{K}_{0}^{\varepsilon}\left(N, g_{N}\right)$ its projection onto the half-spinors bundle $\Sigma_{\varepsilon} N$. Then, the map

$$
\begin{cases}\mathcal{K}_{0}^{\varepsilon}\left(N, g_{N}\right) \rightarrow\left\{\frac{i \varepsilon}{2} \text {-Killing spinors on } \tilde{M}\right\}, \varphi \mapsto e^{\frac{t}{2}} \varphi & \text { if } n \text { is even, } \\ \mathcal{K}_{0}\left(N, g_{N}\right) \rightarrow\left\{\frac{i \varepsilon}{2} \text {-Killing spinors on } \widetilde{M}\right\}, \varphi \mapsto e^{\frac{t}{2}}\left(\varphi \oplus \varepsilon i \frac{\partial}{\partial t} \cdot \varphi\right) & \text { if } n \text { is odd, }\end{cases}
$$

is a well-defined monomorphism. Moreover, if $N$ is compact, then this is actually an isomorphism.

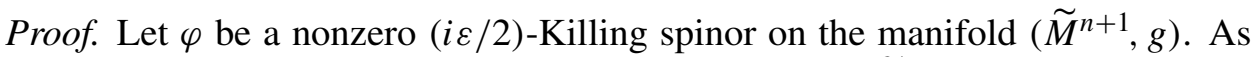
Baum showed (see [1989b] and references therein), if $(\widetilde{M}, g)$ is not isometric to the hyperbolic space, then there must exist a unit smooth vector field $\xi$ on $\widetilde{M}$ with $i \xi \cdot \varphi=\varepsilon \varphi$ on $\widetilde{M}$. From this relationship, the foliated structure of $\widetilde{M}$ can be deduced as follows. First note that $\xi=(\varepsilon V) /|V|$, where $g(V, X):=i\langle X \cdot \varphi, \varphi\rangle$ for all $X \in \Gamma(T \widetilde{M})$, and, in particular, $V=\varepsilon \nabla|\varphi|^{2}$ has no zeros on $\widetilde{M}$. Since $\widehat{\nabla}_{X} V=\varepsilon|\varphi|^{2} X$ (that is $V$ is a closed conformal vector field on $\tilde{M}$ ), one deduces that $\widehat{\nabla}_{X} \xi=X-g(X, \xi) \xi$ for all $X \in \Gamma(T \tilde{M})$, and as a consequence the flow of $\xi-$ which is well-defined and complete since $(\widetilde{M}, g)$ is complete - preserves the level hypersurfaces of $|\varphi|^{2}=|V|$. On the other hand, the second fundamental form of each such hypersurface with respect to $\xi$ is $-\mathrm{Id}$, the Lie derivative of the metric in the direction of $\xi$ is given by $\mathcal{L}_{\xi} g=\left.2 g\right|_{\xi^{\perp} \times \xi^{\perp}}$, and hence, setting

$$
N:=\left\{x \in \tilde{M}:|\varphi|^{2}(x)=1\right\} \subset \tilde{M},
$$

the flow of $\xi$ provides a diffeomorphism $\mathbb{R} \times N \rightarrow \widetilde{M}$ identifying $\xi$ with $\frac{\partial}{\partial t}$ and pulling back the metric $g$ onto $d t^{2} \oplus e^{2 t} g_{N}$, where $g_{N}$ is the metric induced from $g$ onto $N$. This done, the spin Gauss formula (9) implies that, for any $X \in \Gamma(T N)$,

$$
\frac{i \varepsilon}{2} X \cdot \varphi=\widehat{\nabla}_{X} \varphi=\nabla_{X}^{\Sigma N} \varphi-\frac{1}{2} X \cdot \xi \cdot \varphi=\nabla_{X}^{\Sigma N} \varphi+\frac{i \varepsilon}{2} X \cdot \varphi,
$$

from which $\left.\nabla^{\Sigma N} \varphi\right|_{N}=0$ follows: the restriction of $\varphi$ onto any level hypersurface of $|\varphi|^{2}$ is a parallel spinor. Here $\nabla^{\Sigma N}$ stands for the spin Levi-Civita connection on $\Sigma:=\left.\Sigma \widetilde{M}\right|_{N}$. When $n$ is even, the condition $i \xi \cdot \varphi=\varepsilon \varphi$ actually implies that $\varphi \in \Gamma\left(\Sigma_{\varepsilon} N\right)$ since $i \xi$. coincides with the Clifford action of the complex volume form of $\left(N, g_{N}\right)$. When $n$ is odd, the spinor $\left.\varphi\right|_{N}$ can be rewritten in the form $\left.\varphi\right|_{N}=\varphi_{0} \oplus \varepsilon i \frac{\partial}{\partial t} \cdot \varphi_{0}$, where $\varphi_{0} \in \Gamma(\Sigma N)$ is parallel. The dependence on $t$ of $\varphi$ is 
easily computed thanks to

$$
\frac{\partial \varphi}{\partial t}=\widehat{\nabla}_{\frac{\partial}{\partial t}} \varphi=\frac{i \varepsilon}{2} \frac{\partial}{\partial t} \cdot \varphi=\frac{1}{2} \varphi,
$$

from which $\varphi(t, \cdot)=e^{\frac{t}{2}} \varphi(0, \cdot)$ follows. This gives the formulas for the above map, which is obviously a right inverse to the "restriction" map

$$
\left\{\begin{array}{lll}
\left\{\frac{i \varepsilon}{2} \text {-Killing spinors on } \tilde{M}\right\} \rightarrow \mathcal{K}_{0}^{\varepsilon}\left(N, g_{N}\right), & \left.\varphi \mapsto \varphi\right|_{\{0\} \times N} & \text { if } n \text { is even, } \\
\left\{\frac{i \varepsilon}{2} \text {-Killing spinors on } \tilde{M}\right\} \rightarrow \mathcal{K}_{0}\left(N, g_{N}\right), & \left.\varphi \mapsto \varphi_{+}\right|_{\{0\} \times N} & \text { if } n \text { is odd. }
\end{array}\right.
$$

In case $N$ is compact, this restriction map is surjective, a remark which is missing in [Baum 1989a]. To establish this, let $\psi$ be any further nonzero ( $i \varepsilon / 2)$-Killing spinor on $\left(\tilde{M}^{n+1}, g\right)$. Again, $\psi$ splits $\left(\widetilde{M}^{n+1}, g\right)$ as a warped product $\left(\mathbb{R} \times P^{n}, d s^{2} \oplus e^{2 s} g_{\Sigma}\right)$, where $\left(P^{n}, g_{P}\right)$ is complete, spin, and carries a nonzero parallel spinor. Now, using [Montiel 1999], the latter splitting must "coincide" (in a sense that is made precise below) with the former. Namely, for all $t \in \mathbb{R}$, the hypersurface $\{t\} \times N$ is a totally umbilical compact hypersurface of $\widetilde{M}$ with constant mean curvature. Therefore, by applying [op. cit., Lemma 4] to the foliation of $\tilde{M}$ induced by $\psi$ (whose leaves are not assumed to be compact), we easily conclude that for each $t \in \mathbb{R}$, there exists an $s \in \mathbb{R}$ such that $\{t\} \times N=\{s\} \times P$; in particular, $P$ itself must be compact. The same argument shows that, for each $s \in \mathbb{R}$, there exists a $t \in \mathbb{R}$ with $\{s\} \times P=\{t\} \times N$. This yields that, if $\Phi: \mathbb{R} \times P \rightarrow \mathbb{R} \times N,(s, x) \mapsto\left(\phi_{1}(s, x), \phi_{N}(s, x)\right)$, is the isometry induced by both splittings, then the component map $\phi_{1}$ already only depends on $s$. By $\Phi^{*}\left(d t^{2} \oplus e^{2 t} g_{N}\right)=d s^{2} \oplus e^{2 s} g_{P}$ and the existence of an inverse map for $\Phi$ of a similar form, one deduces on the one hand that $\frac{\partial \phi_{N}}{\partial s}(s, x)=0$ and hence $\left(\phi_{1}^{\prime}(s)\right)^{2}=1$ for all $s \in \mathbb{R}$, and on the other hand that $e^{2 s} g_{P}=e^{2 \phi_{1}(s)}\left(\phi_{N}\right)^{*} g_{N}$ holds for all $s \in \mathbb{R}$. This in turn implies the existence of an $s_{0} \in \mathbb{R}$ with $\phi_{1}(s)=s-s_{0}$ and $g_{P}=e^{-2 s_{0}}\left(\phi_{N}\right)^{*} g_{N}$. Thus, up to homotheties on the metrics $g_{P}$ and $g_{N}$, the Riemannian manifolds $\left(P, g_{P}\right)$ and $\left(N, g_{N}\right)$ are isometric and, up to translations in $s$, the splittings $\mathbb{R} \times P$ and $\mathbb{R} \times N$ coincide. By the first part of the proof, $\psi$ must come from a parallel spinor on $N$ and hence lie in the image of the map of Proposition 4.6. This concludes the proof.

From the previous result, we deduce a characterization of hypersurfaces for which inequality (17) is an equality when $\tilde{M}$ is a pseudohyperbolic space in several situations. In fact, as we will see, we are left with the case $n$ is even, the manifold $\left(N^{n}, g_{N}\right)$ has only positive (or only negative) nonzero parallel spinors, and $M$ is only immersed in $\widetilde{M}$. Indeed:

Corollary 4.7. Let $\left(\tilde{M}^{n+1}, g\right):=\left(\mathbb{R} \times N, d t^{2} \oplus e^{2 t} g_{N}\right)$, where $\left(N^{n}, g_{N}\right)$ is a closed nonflat Riemannian spin manifold endowed with at least one nonzero parallel spinor and assume that $\tilde{M}$ carries the induced spin structure (in particular, $(\tilde{M}, g)$ admits an imaginary Killing spinor for at least one of the constants $\left.\pm \frac{i}{2}\right)$. Let $M^{n} \hookrightarrow \widetilde{M}$ 
be any immersed closed orientable hypersurface carrying the induced metric and spin structure and suppose that one of the following supplementary conditions is fulfilled:

(a) $n$ is odd.

(b) $n$ is even and $\left(N^{n}, g_{N}\right)$ has nonzero positive and negative parallel spinors.

(c) $n$ is even and $M^{n}$ bounds a domain in $\tilde{M}$.

Then, $M$ satisfies the equality case in (17) (and so in (7)) if and only if $M=\{t\} \times N$ for some $t \in \mathbb{R}$.

Proof. From Proposition 3.5, if $M^{n} \hookrightarrow \widetilde{M}^{n+1}$ satisfies the equality case in (17), then its mean curvature $H$ must be constant. If either (a) or (b) is fulfilled, then by Proposition 4.6, the manifold $(\tilde{M}, g)$ admits nonzero imaginary Killing spinors for both constants $\pm \frac{i}{2}$, therefore Proposition 3.5 implies that $M$ is totally umbilical which, combined with [Montiel 1999, Lemma 4], yields $M=\{t\} \times N$ for some $t \in \mathbb{R}$. If (c) is fulfilled, this time [Montiel 1999, Theorem 10] applies and yields again $M=\{t\} \times N$ for some $t \in \mathbb{R}$. This shows the "only if" part of the corollary. The "if" part is easy to see since $\lambda_{1}\left(D_{M}\right)=0$ because of parallel spinors on $N$, and on the other hand $|H|=1$ by the explicit form of the metric.

\section{Acknowledgments}

Part of this work was carried out during the Research in Pairs no. 998 at the Centre International de Rencontres Mathématiques (Luminy, France), which Ginoux and Habib would like to thank for their generous support and friendly welcome. Discussions at a latter stage of the paper were made possible thanks to a travel grant awarded to Ginoux by the German Academic Exchange Service (DAAD) and the hospitality of the Lebanese University at Beirut, whom Ginoux would like to thank.

\section{References}

[Ammann and Jammes 2012] B. Ammann and P. Jammes, "The supremum of first eigenvalues of conformally covariant operators in a conformal class", pp. 1-23 in Variational problems in differential geometry, edited by R. Bielawski et al., London Math. Soc. Lecture Note Ser. 394, Cambridge Univ. Press, 2012. MR 2882767 Zbl 1243.53070

[Bär 1998] C. Bär, "Extrinsic bounds for eigenvalues of the Dirac operator", Ann. Global Anal. Geom. 16:6 (1998), 573-596. MR 99k:58183 Zbl 0921.58065

[Bär et al. 2005] C. Bär, P. Gauduchon, and A. Moroianu, "Generalized cylinders in semi-Riemannian and Spin geometry", Math. Z. 249:3 (2005), 545-580. MR 2006g:53065 Zbl 1068.53030

[Baum 1989a] H. Baum, "Complete Riemannian manifolds with imaginary Killing spinors", Ann. Global Anal. Geom. 7:3 (1989), 205-226. MR 91k:58130 Zbl 0694.53043

[Baum 1989b] H. Baum, "Odd-dimensional Riemannian manifolds with imaginary Killing spinors", Ann. Global Anal. Geom. 7:2 (1989), 141-153. MR 91b:53055 Zbl 0708.53039 
[Baum et al. 1991] H. Baum, T. Friedrich, R. Grunewald, and I. Kath, Twistors and Killing spinors on Riemannian manifolds, Teubner-Texte zur Mathematik 124, Teubner, Stuttgart, 1991. MR 94a:53077 Zbl 0734.53003

[Bourguignon and Gauduchon 1992] J.-P. Bourguignon and P. Gauduchon, "Spineurs, opérateurs de Dirac et variations de métriques", Comm. Math. Phys. 144:3 (1992), 581-599. MR 93h:58164 Zbl 0755.53009

[El Soufi and Ilias 1992] A. El Soufi and S. Ilias, "Une inégalité du type "Reilly" pour les sousvariétés de l'espace hyperbolique”, Comment. Math. Helv. 67:2 (1992), 167-181. MR 93i:53059 Zbl 0758.53029

[Friedrich 2000] T. Friedrich, Dirac operators in Riemannian geometry, Graduate Studies in Mathematics 25, Amer. Math. Soc., Providence, RI, 2000. MR 2001c:58017 Zbl 0949.58032

[Ginoux 2002] N. Ginoux, "Reilly-type spinorial inequalities", Math. Z. 241:3 (2002), 513-525. MR 2004a:58037 Zbl 1033.58025

[Ginoux 2003a] N. Ginoux, "Remarques sur le spectre de l'opérateur de Dirac", C. R. Math. Acad. Sci. Paris 337:1 (2003), 53-56. MR 2004h:58046 Zbl 1054.58021

[Ginoux 2003b] N. Ginoux, "Une nouvelle estimation extrinsèque du spectre de l'opérateur de Dirac", C. R. Math. Acad. Sci. Paris 336:10 (2003), 829-832. MR 2004h:58045 Zbl 1054.58020

[Ginoux 2008] N. Ginoux, "The spectrum of the Dirac operator on $\mathrm{SU}_{2} / \mathrm{Q}_{8}$ ", Manuscripta Math. 125:3 (2008), 383-409. MR 2008m:53116 Zbl 1143.53044

[Ginoux 2009] N. Ginoux, The Dirac spectrum, Lecture Notes in Mathematics 1976, Springer, Berlin, 2009. MR 2010a:58039 Zbl 1186.58020

[Heintze 1988] E. Heintze, "Extrinsic upper bounds for $\lambda_{1}$ ”, Math. Ann. 280:3 (1988), 389-402. MR 89f:53091 Zbl 0628.53044

[Hijazi 1986] O. Hijazi, "A conformal lower bound for the smallest eigenvalue of the Dirac operator and Killing spinors”, Comm. Math. Phys. 104:1 (1986), 151-162. MR 87j:58096 Zbl 0593.58040

[Hijazi and Montiel 2013] O. Hijazi and S. Montiel, "A spinorial characterization of hyperspheres", Calc. Var. Partial Differential Equations 48:3-4 (2013), 527-544. MR 3116021 Zbl 1286.53057

[Hitchin 1974] N. Hitchin, "Harmonic spinors", Advances in Math. 14 (1974), 1-55. MR 50 \#11332 Zbl 0284.58016

[Kato 1995] T. Kato, Perturbation theory for linear operators, Classics in Mathematics, Springer, Berlin, 1995. MR 96a:47025 Zbl 0836.47009

[Kühnel and Rademacher 1998] W. Kühnel and H.-B. Rademacher, "Asymptotically Euclidean manifolds and twistor spinors", Comm. Math. Phys. 196:1 (1998), 67-76. MR 99h:53060 Zbl 0929.53023

[Lawson and Michelsohn 1989] H. B. Lawson, Jr. and M.-L. Michelsohn, Spin geometry, Princeton Mathematical Series 38, Princeton Univ. Press, 1989. MR 91g:53001 Zbl 0688.57001

[Montiel 1999] S. Montiel, "Unicity of constant mean curvature hypersurfaces in some Riemannian manifolds", Indiana Univ. Math. J. 48:2 (1999), 711-748. MR 2001f:53131 Zbl 0973.53048

[Reilly 1977] R. C. Reilly, "On the first eigenvalue of the Laplacian for compact submanifolds of Euclidean space”, Comment. Math. Helv. 52:4 (1977), 525-533. MR 58 \#2657 Zbl 0382.53038

[Setti 1998] A. G. Setti, "Eigenvalue estimates for the weighted Laplacian on a Riemannian manifold", Rend. Sem. Mat. Univ. Padova 100 (1998), 27-55. MR 2000a:58082 Zbl 0922.58084 
Received March 19, 2014.

NiCOLAS GINOUX

DÉPARTEMENT INFORMATIQUE

IUT DE METZ

UNIVERSITÉ DE LORRAINE

ILE DU SAULCY

CS 10628

57045 METZ

FRANCE

nicolas.ginoux@univ-lorraine.fr

GEORGES HABIB

FACULTY OF SCIENCES II

DEPARTMENT OF MATHEMATICS

LEBANESE UNIVERSITY

P.O. BOX 90656

FANAR-MATN

LEBANON

ghabib@ul.edu.lb

SIMON RAULOT

LABORATOIRE DE MATHÉMATIQUES R. SALEM UMR 6085 CNRS

UNIVERSITÉ DE ROUEN AVENUE DE L'UNIVERSITÉ

BP.12 TECHNOPÔLE DU MADRILLET

76801 SAINT-ÉTIENNE-DU-ROUVRAY

FRANCE

simon.raulot@univ-rouen.fr 


\title{
PACIFIC JOURNAL OF MATHEMATICS
}

\author{
msp.org/pjm
}

Founded in 1951 by E. F. Beckenbach (1906-1982) and F. Wolf (1904-1989)

\section{EDITORS}

Don Blasius (Managing Editor)

Department of Mathematics

University of California

Los Angeles, CA 90095-1555

blasius@math.ucla.edu

\author{
Paul Balmer \\ Department of Mathematics \\ University of California \\ Los Angeles, CA 90095-1555 \\ balmer@math.ucla.edu \\ Robert Finn \\ Department of Mathematics \\ Stanford University \\ Stanford, CA 94305-2125 \\ finn@math.stanford.edu \\ Sorin Popa \\ Department of Mathematics \\ University of California \\ Los Angeles, CA 90095-1555 \\ popa@math.ucla.edu
}

\author{
Vyjayanthi Chari \\ Department of Mathematics \\ University of California \\ Riverside, CA 92521-0135 \\ chari@math.ucr.edu \\ Kefeng Liu \\ Department of Mathematics \\ University of California \\ Los Angeles, CA 90095-1555 \\ liu@math.ucla.edu \\ Jie Qing \\ Department of Mathematics \\ University of California \\ Santa Cruz, CA 95064 \\ qing@ cats.ucsc.edu
}

\section{PRODUCTION}

Silvio Levy, Scientific Editor, production@msp.org

\section{SUPPORTING INSTITUTIONS}

ACADEMIA SINICA, TAIPEI

CALIFORNIA INST. OF TECHNOLOGY

INST. DE MATEMÁTICA PURA E APLICADA

KEIO UNIVERSITY

MATH. SCIENCES RESEARCH INSTITUTE

NEW MEXICO STATE UNIV.

OREGON STATE UNIV.

\author{
STANFORD UNIVERSITY \\ UNIV. OF BRITISH COLUMBIA \\ UNIV. OF CALIFORNIA, BERKELEY \\ UNIV. OF CALIFORNIA, DAVIS \\ UNIV. OF CALIFORNIA, LOS ANGELES \\ UNIV. OF CALIFORNIA, RIVERSIDE \\ UNIV. OF CALIFORNIA, SAN DIEGO \\ UNIV. OF CALIF., SANTA BARBARA
}

\author{
Daryl Cooper \\ Department of Mathematics \\ University of California \\ Santa Barbara, CA 93106-3080 \\ cooper@math.ucsb.edu \\ Jiang-Hua Lu \\ Department of Mathematics \\ The University of Hong Kong \\ Pokfulam Rd., Hong Kong \\ jhlu@maths.hku.hk \\ Paul Yang \\ Department of Mathematics \\ Princeton University \\ Princeton NJ 08544-1000 \\ yang@math.princeton.edu
}

These supporting institutions contribute to the cost of publication of this Journal, but they are not owners or publishers and have no responsibility for its contents or policies.

See inside back cover or msp.org/pjm for submission instructions.

The subscription price for 2015 is US \$420/year for the electronic version, and \$570/year for print and electronic.

Subscriptions, requests for back issues and changes of subscribers address should be sent to Pacific Journal of Mathematics, P.O. Box 4163, Berkeley, CA 94704-0163, U.S.A. The Pacific Journal of Mathematics is indexed by Mathematical Reviews, Zentralblatt MATH, PASCAL CNRS Index, Referativnyi Zhurnal, Current Mathematical Publications and Web of Knowledge (Science Citation Index).

The Pacific Journal of Mathematics (ISSN 0030-8730) at the University of California, c/o Department of Mathematics, 798 Evans Hall \#3840, Berkeley, CA 94720-3840, is published twelve times a year. Periodical rate postage paid at Berkeley, CA 94704, and additional mailing offices. POSTMASTER: send address changes to Pacific Journal of Mathematics, P.O. Box 4163, Berkeley, CA 94704-0163.

PJM peer review and production are managed by EditFLOW ${ }^{\circledR}$ from Mathematical Sciences Publishers.

\section{PUBLISHED BY}

\section{mathematical sciences publishers \\ nonprofit scientific publishing}

http://msp.org/

(C) 2015 Mathematical Sciences Publishers 


\section{PACIFIC JOURNAL OF MATHEMATICS}

Volume $278 \quad$ No. $1 \quad$ November 2015

Growth tight actions

Goulnara N. Arzhantseva, Christopher H. Cashen and JING TAO

A flag structure on a cusped hyperbolic 3-manifold

ELISHA FALBEL and RAFAEL SANTOS THEBALDI

A new upper bound for the Dirac operators on hypersurfaces

Nicolas GinouX, GeORGES Habib and Simon RaUlot

Games and elementary equivalence of $\mathrm{II}_{1}$-factors

ISAAC GOLDBRING and THOMAS SINCLAIR

Grossberg-Karshon twisted cubes and hesitant walk avoidance

MEGUMI HARADA and EUNJEONG LEE

Gamma factors of distinguished representations of $\mathrm{GL}_{n}(\mathbb{C})$

ALEXANDER KEMARSKY

The $W$-entropy formula for the Witten Laplacian on manifolds with 173 time dependent metrics and potentials

SONGZI LI and XIANG-DONG LI

A diagrammatic categorification of the affine $q$-Schur algebra $\hat{\boldsymbol{S}}(n, n) 201$ for $n \geq 3$

MARCO MACKAAY and ANNE-LAURE THIEL

Showing distinctness of surface links by taking 2-dimensional braids

INASA NAKAMURA

Correction to Modular $L$-values of cubic level 